\title{
Computer-vision-based technology for fast, accurate and cost effective diagnosis of malaria
}

\author{
Bina Srivastava', Anupkumar R. Anvikar1*, Susanta K. Ghosh², Neelima Mishra', Navin Kumar', \\ Arnon Houri-Yafin ${ }^{3}$, Joseph Joel Pollak ${ }^{3}$, Seth J. Salpeter ${ }^{3}$ and Neena Valecha ${ }^{1}$
}

\begin{abstract}
Background: Microscopy has long been considered to be the gold standard for diagnosis of malaria despite the introduction of newer assays. However, it has many challenges like requirement of trained microscopists and logistic issues. A vision based device that can diagnose malaria, provide speciation and estimate parasitaemia was evaluated.

Methods: The device was evaluated using samples from 431 consented patients, 361 of which were initially screened by RDT and microscopy and later analysed by PCR. It was a prospective, non-randomized, blinded trial. Quantification of parasitaemia was performed by two experienced technicians. Samples were subjected to diagnosis by Sight Dx digital imaging scanning.

Results: The sensitivity and specificity of the SightDx P1 device for analysed samples were found to be 97.05 and $96.33 \%$, respectively, when compared to PCR. When compared to microscopy, sensitivity and specificity were found to be 94.4 and $95.6 \%$, respectively. The device was able to speciate $73.3 \%$ of the PCR Plasmodium falciparum positive samples and $91.4 \%$ of PCR Plasmodium vivax positive samples.
\end{abstract}

Conclusion: The ability of the device to detect parasitaemia as compared with microscopy, was within $50 \%$ in $71.3 \%$ of cases and demonstrated a correlation coefficient of 0.89 .

Keywords: Malaria, Diagnosis, PCR, Device, Sensitivity, Specificity

\section{Background}

According to the World Health Organization (WHO) estimates, there were $\sim 207$ million cases and 627,000 deaths due to malaria in 2012 [1]. Half of the world's population is at risk of being infected by malaria. Definitive diagnosis of malaria is imperative for rapid treatment, reducing morbidity and mortality, preventing unnecessary use of anti-malarials and increased drug resistance [2]. As a result, the WHO now recommends that all cases of suspected malaria should be confirmed using malaria diagnostic tests prior to treatment. Microscopy continues to be the gold standard for diagnosis of malaria [2]

\footnotetext{
*Correspondence: anvikar@rediffmail.com

${ }^{1}$ National Institute of Malaria Research, Sector 8 Dwarka, New Delhi 110 077, India

Full list of author information is available at the end of the article
}

but is time and labour intensive, requires qualified personnel, and is subjective. Rapid diagnostic tests (RDTs) have revolutionized the diagnosis of malaria, particularly in remote areas [3] but suffer from difficulties in transport and storage, persistence of Hrp-2 in the blood, poor detection at very low parasitaemia, and inability to quantify the parasites. Other techniques available for diagnosis include immunologic techniques, Polymerase Chain Reaction (PCR), Loop-mediated isothermal amplification (LAMP), all of which present unique challenges. PCR has potential to detect low parasitaemia but is expensive, time consuming, and requires highly trained personnel. LAMP is being evaluated $[4,5]$ and is still in early stages of development [6].

The SightDx P1 Device aims to overcome these deficits: the computer-vision-based technology is designed for fast, accurate and cost effective diagnosis of malaria in 
blood samples. Additionally the device is able to rapidly report parasitaemia levels after scanning over 1 million red blood cells.

\section{Methods}

This trial aimed to evaluate the efficacy of the device, as measured by the sensitivity and specificity at different levels of parasitaemia. The device was evaluated on 431 consented patients. Sensitivity and specificity of the device were compared against PCR and microscopy. Rapid diagnostic tests (RDTs) were used for initial patient screening and were also compared against ground truth. All patients were symptomatic. The primary endpoint was to assess the sensitivity and specificity of the device using PCR as the gold standard. The secondary endpoints were species identification, accuracy of parasitaemia estimation and comparison with microscopy.

\section{Study design}

This was a single center, prospective, non-randomized, blinded trial. All technicians were blinded to the results of each branch of the study. Comparison between study outcomes was done only after data collection and the examination was complete.

\section{Study procedures}

All patients presenting with fever at the NIMR clinic were considered eligible for enrollment and were given the option to participate. Informed consent was obtained prior to sample collection. Suspected patients for Plasmodium vivax and Plasmodium falciparum were routinely diagnosed in the malaria clinic of Wenlock Hospital, Mangalore, by examining Giemsa-stained slides (and/or malaria RDTs as routinely used in the clinic; SD Malaria $\mathrm{Ag} \mathrm{Pf} / \mathrm{Pv}$ in this case). Malaria treatment was solely based on the malaria clinic diagnosis process and patients' course of treatment [7] was not changed due to the Parasite P1 device or by the NIMR microscopy diagnoses. In parallel, about $4 \mu \mathrm{L}$ of blood was collected in microfuge tube containing EDTA. These samples were scanned onsite by using the P1 device, while P1 final analysis was conducted blindly in SightDx's R\&D facility in Israel. In addition, blood was also collected on filter paper spots for PCR evaluation at NIMR, and was used for the preparation of two Giemsa-stained smears for microscopic examination at NIMR. The Giemsa-stained smears and filter papers were transported to NIMR, New Delhi.

The Giemsa slides were reviewed by a NIMR microscopist at Wenlock NIMR clinic in Mangalore, and by a second microscopist at the NIMR laboratory in New Delhi. If there was disagreement between the two reads, or a discrepancy in parasitaemia level of greater than $50 \%$, a third microscopist reviewed the slide and made a final determination.

Diagnostic PCR assays were carried out at NIMR. For this, DNA was isolated from filter paper using the QIAamp mini kit (51306) according to the manufacturer's instructions and stored at $-20{ }^{\circ} \mathrm{C}$ until PCR could be completed. Nested PCR was performed with primers described previously [7, 8]. Known positive sample from parasite bank of NIMR and previously uninfected individual's blood was used as positive and negative control, respectively.

The SightDx digital imaging scanning was performed onsite. A blood droplet was stained and diluted in a proprietary solution. This diluted sample was placed inside a flow-cell disposable slide. The slide was then loaded into the P1 machine where it was scanned automatically. Each field was autofocused and stained covering a total of 270 fields corresponding to $0.2 \mu \mathrm{l}$ of blood. The complete scan took less than 5 min per sample. At peak participant volume, 56 patients were analysed in an $8 \mathrm{~h}$ working day.

Computer vision and statistical models were used to detect the malaria parasites. The algorithm used fluorescent cues to detect hotspots and then classified these into white blood cells, parasites, or "other". The algorithm also estimated RBC density. Using statistical models, SightDx P1 determined infection status, parasitaemia levels, and species. Diagnosis and parasitaemia are statistical constructs, and there is an Internal Calibration Parameter that determines whether a sample is considered "negative" that can be adjusted to optimize the tradeoff between sensitivity and specificity for the use at hand (e.g. screening or confirmation).

\section{Statistical methods}

Sensitivity and specificity analysis and $95 \%$ confidence intervals (CIs) were computed using a $2 \times 2$ table for outcomes of the tested device and the reference outcome. Test sensitivity (conditional probability that the test is positive if the condition is positive), calculated by the following formula:

$$
\begin{aligned}
\text { Sensitivity }= & (\text { True Positive }) / \\
& (\text { True Positive }+ \text { False Negative }) \times 100
\end{aligned}
$$

Test specificity (conditional probability that the test is normal if the condition is normal (negative), calculated by the following formula:

$$
\begin{aligned}
\text { Specificity }= & (\text { True Negative }) / \\
& (\text { True Negative }+ \text { False Positive }) \times 100
\end{aligned}
$$

Kappa Coefficients were calculated for analysing the agreement between the diagnoses of the two 
microscopists. The data was analysed using the SAS ${ }^{\circledR}$ version 9.1 (SAS Institute, Cary North Carolina).

\section{Results}

The SightDx P1 device is a tabletop device for malaria diagnostics (Fig. 1). Cartridges are placed in the device, scanned and output is presented on the device touch screen.

The evaluation of SightDx P1 device performance was based on 431 febrile patients reporting to the Malaria Clinic at Wenlock Hospital, Mangalore and consenting to participate. Figure 2 shows the description of the evaluable samples. Of 431 patients consented to participate, 70 samples were not included in the analysis for various reasons: operator errors, such as slide placement error, insufficient filling or overfilling of the slide $(\mathrm{n}=22)$, external technical problems, such as interruptions during running of the test and power failure $(\mathrm{n}=3)$, missing PCR results $(\mathrm{n}=5)$ and samples marked as undecided by the automated algorithm $(\mathrm{n}=40)$, which need further investigation. These 40 samples will henceforth be referred to as 'flagged' samples in this article. Thus, the study's population involved 361 samples that were evaluated by SightDx device, PCR and microscopy.

\section{SightDx compared to PCR and microscopy}

The device's sensitivity and specificity, when compared to PCR, were found to be $97.05 \%$ (95\% CI 94.5-99.6) and 96.33\% (95\% CI 93.7-99.0), respectively.

SightDx sensitivity and specificity compared to microscopy were found to be $94.4 \%$ (95 \% CI 90.997.8) and $95.6 \%$ (95\% CI 92.7-98.6), respectively (Table 1).

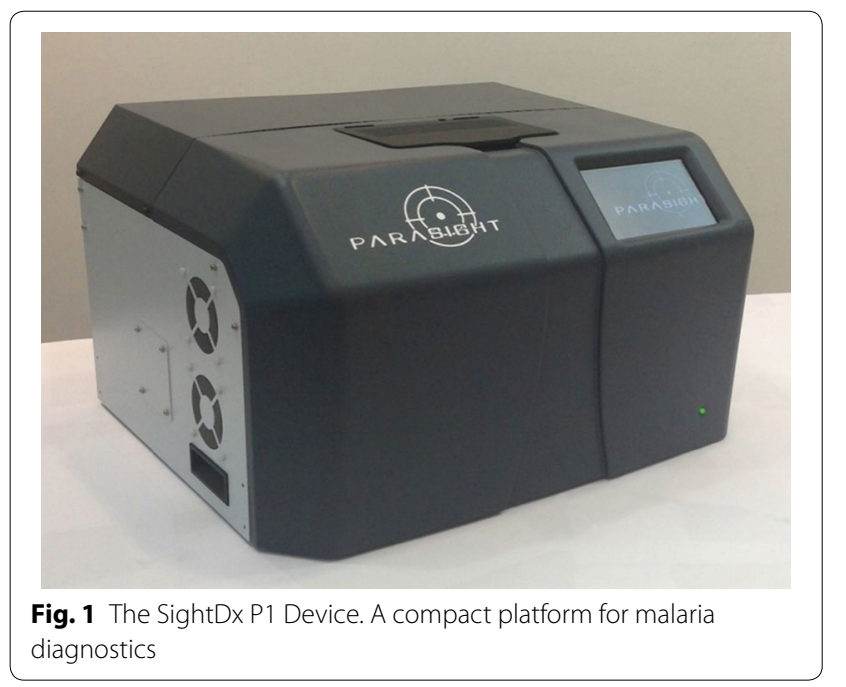

\section{RDT compared to PCR and microscopy}

The RDT's sensitivity and specificity, when compared to PCR, were found to be $93.5 \%$ (95 \% CI 89.8-97.2) and $96.3 \%$ (95 \% CI 93.7-99.0), respectively. Sensitivity and specificity of the RDT compared to microscopy were found to be $94.3 \%$ (95\% CI 90.0-97.8) and $96.7 \%$ (95\% CI 94.2-99.3), respectively (Table 2).

\section{Speciation}

The ability of the device to identify two types of malaria species was compared with PCR. The current version of the device did not support mixed infection reporting. From samples found to be positive by both PCR and SightDx, SightDx speciated $73.3 \%$ of PCR P. falciparum + samples and $91.4 \%$ of PCR P. vivax + samples, as shown in Table 3. Notably, all of the mixed infection samples were positively detected by SightDx as infected. Eleven mixed infections were reported as $P$. vivax, two were reported as $P$. falciparum, while one was reported positive.

\section{Parasitaemia}

To correlate the microscopists analysis with the parasitaemia provided by the SightDx device Pearsons correlation and Spearmans correlation analysis was carried out. Spearman's rank correlation coefficient analysis is preferred for monotonic relationships. The Spearman's rank correlation coefficient and the Pearsons correlation coefficient calculated when comparing SightDx's parasitaemia reporting to microscopy on infected samples. Following the WHO criteria, SightDx was analysed on whether or not it produced parasitaemia levels of $\pm 50 \%$ of microscopy. Microscopy parasitaemia was considered as the average of the two microscopists' estimation, or the third microscopist's estimation in cases of disagreement. SightDx was within $50 \%$ in $71.3 \%$ of cases (in 117 out of 164). The overall correlation between the two data sets can be seen in Fig. 3.

\section{Discussion}

The study evaluates a novel platform for diagnosing malaria. It showed sensitivity of $97.5 \%$ and specificity of $96.33 \%$ when compared to PCR. Moreover, the study showed that the platform provides high sensitivity in cases of low parasitaemia. Going by the WHO standard of $95 \%$ sensitivity for malaria diagnosis, the device has excellent sensitivity. This is the first example of a complete vision based malaria diagnostic platform, as attempts to develop vision-based device have been made in the past with limited success [9-12].

The device was able to speciate $73.3 \%$ of the P. falciparum positive samples and $91.4 \%$ of $P$. vivax positive samples compared to PCR. However currently it does not 


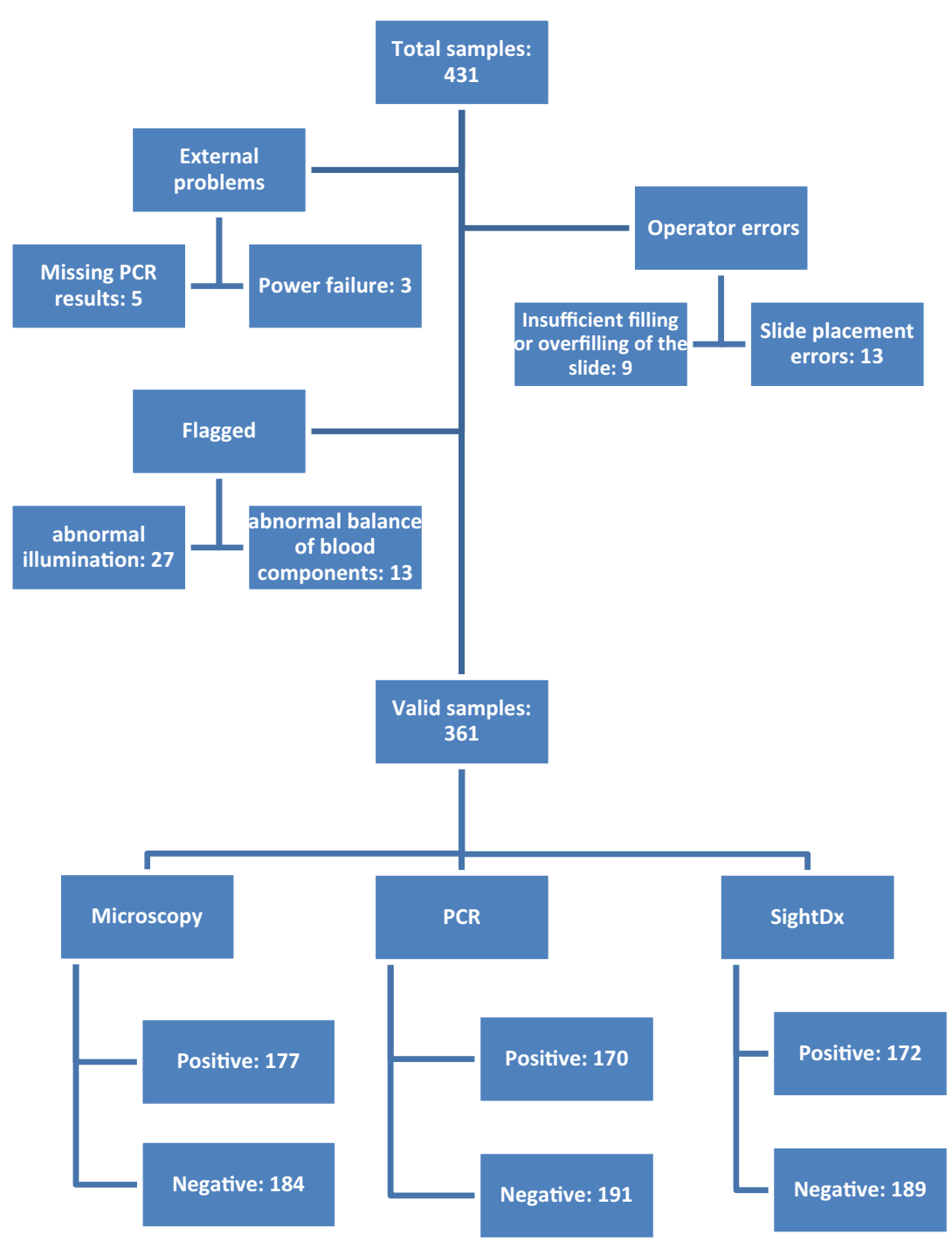

Fig. 2 Description of the samples. A total of 431 samples were collected and 361 were analyzed. Removed samples were due to technical errors or due to flagged samples

Table 1 Sensitivity and specificity of P1 device versus PCR and microscopy

\begin{tabular}{|c|c|c|c|c|c|c|}
\hline \multirow[t]{2}{*}{ Comparator } & \multicolumn{3}{|c|}{ Sensitivity } & \multicolumn{3}{|c|}{ Specificity } \\
\hline & Percent & $n / N$ & $95 \% \mathrm{Cl}$ & Percent & $n / N$ & $95 \% \mathrm{Cl}$ \\
\hline Microscopy & 94.4 & $167 / 177$ & $90.9-97.8$ & 95.6 & $176 / 184$ & $92.7-98.6$ \\
\hline$P C R$ & 97.05 & $165 / 170$ & $94.5-99.6$ & 96.33 & 184/191 & $93.7-99.0$ \\
\hline
\end{tabular}

Table 2 Sensitivity and specificity of RDT versus PCR and microscopy

\begin{tabular}{|c|c|c|c|c|c|c|}
\hline \multirow[t]{2}{*}{ Comparator } & \multicolumn{3}{|c|}{ Sensitivity } & \multicolumn{3}{|c|}{ Specificity } \\
\hline & Percent & $n / N$ & $95 \% \mathrm{Cl}$ & Percent & $n / N$ & $95 \% \mathrm{Cl}$ \\
\hline Microscopy & 94.3 & $167 / 177$ & $90.9-97.8$ & 96.7 & $178 / 184$ & $94.2-99.3$ \\
\hline$P C R$ & 93.5 & $159 / 170$ & $89.8-97.2$ & 96.3 & 184/191 & $93.7-99.0$ \\
\hline
\end{tabular}


Table 3 Speciation accuracy divided according to treatment groups

\begin{tabular}{llll}
\hline & SightDx accuracy (\%) & In numbers & $\mathbf{9 5} \% \mathbf{C l}$ \\
\hline P. vivax & 91.4 & $96 / 105$ & $86.1-96.8$ \\
P. falciparum & 73.3 & $34 / 46$ & $61.2-86.6$ \\
Mixed infection & 0 & $0 / 14$ & $\mathrm{NA}$ \\
\hline
\end{tabular}

detect mixed samples since a large enough database has not been collected. There is scope of improvement in this area since there are many countries having both the species and also have good significant proportion of mixed infections [13].

Enumeration of parasites in malaria patients is extremely important to know the severity of malaria, as a prognostic indicator, drug efficacy studies and clinical trials. Despite availability of various tools for malaria diagnosis, microscopy is the mainstay for quantitation of parasites. Though accurate, easy to perform, cost effective, it is labor intensive and can vary between technicians. Very few tools are able to estimate the parasitaemia in malaria patients. Real time PCR has the potential to quantify parasites and has been proved to be useful [14]. Flow cytometry could also accurately measure parasitaemia in rodent malaria $[9,10]$. Nonetheless, these methods need sophisticated equipment and are not cost effective. The SightDx P1 platform has a very powerful use for quantitative estimation of parasitaemia. It could detect the parasite count within $50 \%$ of that detected by microscopy in $71.3 \%$ of the cases. Most notably, the device demonstrated a correlation coefficient of 0.89 demonstrating a strong statistical similarity, and convincingly showing the relevance of the technology in clinical malaria assessment.

Strikingly, the test demonstrated significantly improved sensitivity and specificity when compared to the RDT used in the study. RDTs are known to have poor sensitivity specifically at low parasitaemia. Moreover, even when positive bands do appear, many times they are weak lines, which are hard to interpret. While RDTs showed a sensitivity of only $93.5 \%$ the P1 platform showed a sensitivity of $97.05 \%$. The SightDx device offers better sensitivity and specificity and clearly states whether the sample is positive or negative, preventing mis-interpretation or difficulties in analysis.

Though the device showed good sensitivity and specificity against PCR, several improvements are still needed. The software version used in this study is not equipped to detect mixed infections, and while all the mixed infection samples were diagnosed as infected by the device, it interpreted them mostly as $P$. vivax. Furthermore, the device is capable of identifying the infection stage of a sample, but verifying the accuracy of infection stage identification was not in the scope of this study. There were operator errors responsible for indeterminate results. Such errors could be eliminated by including a modified sample-loading mechanism and improved sample-preparation procedures. The operator errors can be identified during the scanning of the sample, in which case, the user can be notified to re-run the sample. There were external conditions that caused test failures. The device could be improved to identify that an interruption has occurred and request the user to re-run the sample.

Some samples could not be analysed due to the reasons discussed in Fig. 2. However, several software and hardware correction steps were implemented since the data collection completion, aiming at dramatically lowering the rejection and undetermined sample rate. These steps include sample and slide preparation steps to reduce overfilling or insufficient slide filling and to improve staining solution consistency, slide holding improvements to reduce slide misplacement, software improvements in handling sample deviation and support for real-time user re-scan report flag.

Among the 70 rejected samples, reference results were available for 64 samples. Three were positive for $P$. falciparum, 11 were $P$. vivax and one was mixed infection. Since the device did not provide results for these samples, they could not be included in the analysis.

CL-IN002 was a single site clinical trial. Multi-site studies will help further validating the device. Beyond India, future tests sites will be placed throughout Africa, South East Asia as well as European areas demonstrating non-endemic malaria infections. A diversity of locations will further strengthen the device database and demonstrate the broad ability of the device to diagnosis a variety of genetic backgrounds.

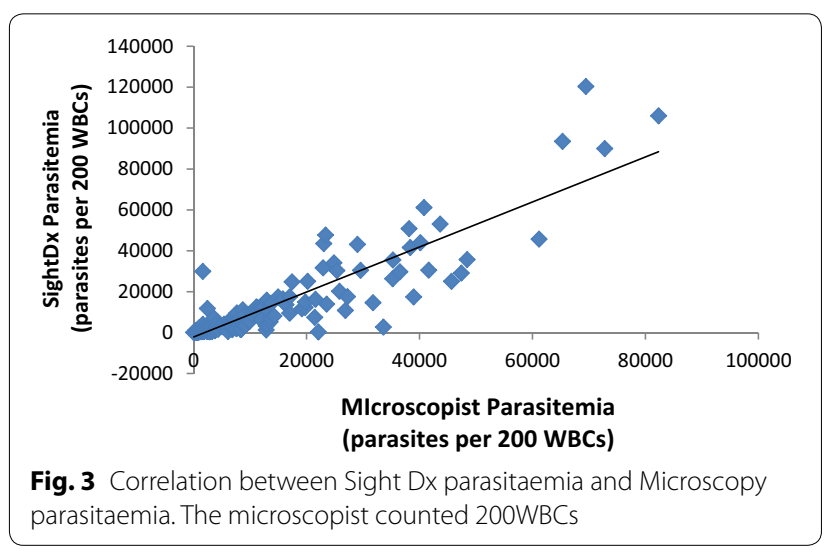




\section{Conclusion}

The SightDx P1 device demonstrated a sensitivity of $97.03 \%$ and a specificity of $96.33 \%$, significantly exceeding the WHO criteria of $95 \%$ accuracy and showing superior performance to RDT. Furthermore, since it can evaluate speciation and estimate the parasitaemia, it can be a valuable tool in clinical practice as well as research studies.

\section{Author's contribution}

AHY, JJP, SJS, ARA, NV, BS were investigators responsible for the design of the project proposal and monitored the progress. BS, SKG, NM were involved in execution of study, data collection and quality check of data, NK involved in molecular studies. ARA, BS, AHY, JJP, SJS compiled and performed statistical analysis. AKA wrote the first draft and SJS and NV corrected the draft. All authors read and approved the final manuscript.

\section{Author details}

${ }^{1}$ National Institute of Malaria Research, Sector 8 Dwarka, New Delhi 110077 , India. ${ }^{2}$ National Institute of Malaria Research Field Unit, Bengaluru, India. ${ }^{3}$ Sight Diagnostics, 1 Agudat Hasport Hapoel, Jerusalem Technology Park, Jerusalem, Israel.

\section{Acknowledgements}

The authors are thankful to the Ministry of Health, Government of India for providing facilities We also thank the patients who agreed to be a part of this study. Acknowledgements are also due to all the staff members of NIMR involved in the study. This study was approved by NIMR Institutional Ethics Committee (letter no ECR/NIMR/EC/2012/58, dated 7 May 2012) while manuscript was approved by the NIMR's Publication Screening Committee (018/2015).

\section{Funding source}

The study was funded by the SightDx Ltd, Jerusalem Technology Park, Israel.

\section{Competing interests}

AHY, JJP and SJS are employed in SightDx Ltd. They developed and also operated the device. They also contributed the methodology part of this manuscript. Other authors have no competing interests.

Received: 13 March 2015 Accepted: 17 December 2015

Published online: 30 December 2015
2. WHO. Guidelines for the treatment of malaria. 2nd ed. Geneva: World Health Organization; 2010. http://www.who.int/malaria/publications/ atoz/9789241547925/enindex.html. Accessed 1 Apr 2014.

3. Yan J, Li N, Wei X, Li P, Zhao Z, Wang L, et al. Performance of two rapid diagnostic tests for malaria diagnosis at the China-Myanmar border area. Malar J. 2013;12:73. doi:10.1186/1475-2875-12-73.

4. Kumar N, Singh JP, Pande V, Mishra N, Srivastava B, Kapoor R, et al. Genetic variation in histidine rich proteins among Indian Plasmodium falciparum population: possible cause of variable sensitivity of malaria rapid diagnostic tests. Malar J. 2012;11:298. doi:10.1186/1475-2875-11-298.

5. Singh R, Savargaonkar D, Bhatt R, Valecha N. Rapid detection of Plasmodium vivax in saliva and blood using loop mediated isothermal amplification (LAMP) assay. J Infect. 2013;67:245-7. doi:10.1016/j.jinf.2013.04.016

\section{(Epub 2013 Apr 25)}

6. Erdman LK, Kain KC. Review molecular diagnostic and surveillance tools for global malaria control. Travel Med Infect Dis. 2008;6:82-99.

7. National Vector Borne Diseases Control Programme. Guidelines for diagnosis and treatment of malaria in India. 2014. http://www.mrcindia.org/ Diagnosis\%20of\%20Malaria\%20pdf/Guidelines\%202014.pdf. Accessed 5 Sept 2015.

8. Snounou G, Viriyakosol S, Jarra W, Pinheiro L, do Rosario VE, Thaithong $S$, et al. High sensitivity of detection of human malaria parasites by the use of nested polymerase chain reaction. Mol Biochem Parasitol. 1993;2:315-20.

9. Johnston SP, Pieniazek NJ, Zayavong MV, Slemenda SB, Wilkins PP, da Silva AJ. PCR as a confirmatory technique for laboratory diagnosis of malaria. J Clin Microbiol. 2006;44:1087-9.

10. Xie L, Li Q, Johnson J, Zhang J, Milhous W, Kyle D. Development and validation of flow cytometric measurement for parasitaemia using autofluorescence and YOYO-1 in rodent malaria. Parasitology. 2007;134:1151-62.

11. Frean JA. Reliable enumeration of malaria parasites in thick blood films using digital image analysis. Malar J. 2009;8:218

12. Prescott WR, Jordan RG, Grobusch MP, Chinchilli VM, Kleinschmidt I, Borovsky J, et al. Performance of a malaria microscopy image analysis slide reading device. Malar J. 2012;11:155.

13. Gupta B, Gupta P, Sharma A, Singh V, Dash AP, Das A. High proportion of mixed-species Plasmodium infections in India revealed by PCR diagnostic assay. Trop Med Int Health. 2010;15:819-24.

14. Andrews L, Andersen RF, Webster D, Dunachie S, Walther RM, Bejon $P$, et al. Quantitative real-time polymerase chain reaction for malaria diagnosis and its use in malaria vaccine clinical trials. Am J Trop Med Hyg. 2005;73:191-8.

\section{References}

1. WHO: World Malaria Report 2012, Geneva: World Health Organization. 2012. http://www.who.int/malaria/media/world_malaria_report_2013/ en/. Accessed 1 April 2014.

\section{Submit your next manuscript to BioMed Central and we will help you at every step: \\ - We accept pre-submission inquiries \\ - Our selector tool helps you to find the most relevant journal \\ - We provide round the clock customer support \\ - Convenient online submission \\ - Thorough peer review \\ - Inclusion in PubMed and all major indexing services \\ - Maximum visibility for your research}

Submit your manuscript at www.biomedcentral.com/submit

\section{(O) Biomed Central}

